This is an Author's Original Manuscript of an article published by Taylor \& Francis in Theology and Science on October 12, 2020, available online: https://doi.org/10.1080/14746700.2020.1825195.

\title{
Theodicy, Supreme Providence, and Semiclassical Theism
}

\author{
by James Goetz \\ https://philpeople.org/profiles/james-goetz \\ james.goetz@ncis.org
}

\begin{abstract}
Logical limits of omnipotence, the problem of evil, and a compelling cosmological argument suggest the position of supreme providence and the foremost creation out of nothing that coheres with the constraints of physics. The Supreme Being possesses everlasting love, perception, and force while governing the universe of probabilistic processes and freewill creatures. For example, the Supreme Being intervenes in the processes of creation by the means of synergism with freewill creatures and cannot meticulously control the created universe.
\end{abstract}

\section{Keywords}

theodicy; problem of evil; divine providence; divine attributes; creation out of nothing; cosmological argument; miracles

\section{Introduction}

That to the highth of this great Argument I may assert Eternal Providence, And justifie the wayes of God to men. ${ }^{1}$

Positions of theodicy propose justified belief in the existence of God despite a world filled with horrific evil. When a theodicy supports the traditional attributes of God, specifically omnipotence, omniscience, and omnibenevolence, then the theodicy proposes a resolution for the problem of evil. By definition, the problem of evil states that the extensive horrific suffering in the world disagrees with justified belief in God who possesses the traditional divine attributes of omnipotence, omniscience, and omnibenevolence.

Consider the problem of evil. Extensive horrific suffering caused by diseases, accidents, and natural disasters could be prevented by God as defined by traditional divine attributes. Also, theists believe that God wants them to protect and help people who suffer from these horrors of nature. The protection and help includes prayers and practical support. So why does God not prevent these horrors of nature or do more to fix the consequences of the horrors? Furthermore, most theists support the moral rightness of protecting society by incarcerating perpetrators of serial rape, serial killing, mass murder, terrorism, human 
trafficking, and all crimes against humanity. So why does God not do more to protect society from the horrors caused by horrific perpetrators?

I am impressed by recent proposals to resolve the problem of evil. For example, skeptical theism proposes that God exists while we should be skeptical of our ability to discern why God permits extensive horrors of nature and horrific crime. ${ }^{2}$ However, the proposals leave me unconvinced mostly because the traditional position of divine omnipotence is unnecessary. For example, consider the logical limits of divine omnipotence introduced in Propositions 1-2:

Proposition 1: God could possibly create a physical universe out of nothing while that universe is beyond meticulous control.

Proposition 2: Proposition 1 implies that God cannot meticulously control all possible universes.

One might argue that traditional theism is unbound by the logical analysis of divine omnipotence, so Propositions 1-2 are irrelevant. However, Aquinas implied the logical consistency of divine omnipotence when he stated that God cannot change the past because the past no longer tangibly exists. ${ }^{3}$ Likewise, traditional theism does not necessarily flout the logic of omnipotence. That said, traditional theism states that God can meticulously control the created universe.

Sanders divides types of traditional Christianity into two top types of divine providence, that is, meticulous providence and general providence. ${ }^{4}$ Meticulous providence implies that God meticulously controls every good and evil event in the universe. General providence is part of freewill theism and implies that God can meticulously control every good and evil event in the universe while God permits creaturely free will without meticulously controlling it. Proponents of meticulous providence include Augustine, Aquinas, Molinists, and Calvinists. Proponents of general providence include many Church Fathers, the Eastern Orthodox Church, Anabaptists, Arminians, Methodists, most open theists, and the majority of Charismatic Christianity.

I define another type of divine providence. For example, process theism (PT) and semiclassical theism (ST) are positions that reject the traditional doctrine of divine omnipotence and state that God exists while possessing the supreme attributes of everlasting love, complete knowledge of the present, and everlasting power. I call this supreme providence because God governs the universe with the supreme attributes. In other words, the Supreme Being lovingly governs the universe and cannot possibly exercise meticulous control over creation. Proponents of PT, also called neoclassical theism, include Hartshorne,${ }^{5}$ Edwards, ${ }^{6}$ Griffin, ${ }^{7}$ Cobb, ${ }^{8}$ and Oord. ${ }^{9}$ Alternatively, Goetz introduced ST. ${ }^{10}$ Related ancient versions of supreme providence include the teachings of providence in Zoroastrianism and Plato's Timaeus. ${ }^{11}$

Among the positions of supreme providence, only ST proposes a foremost creation out of nothing. ${ }^{12}$ Likewise, ST is the only position of supreme providence that coheres with any cosmological argument that implies the existence of the Supreme Being and a model for the foremost creation from nothing. Also, 
theodicy and a compelling cosmological argument support each other. For example, why bother with theodicy if there is no compelling evidence to support the necessary existence of God? Or why care about the necessary existence of God if God is unhelpful or unloving? However, evidence that God is a necessary being who possesses everlasting love and help has extraordinary value.

One might wonder how God's love can be helpful if God cannot meticulously control the universe or prevent extensive horrific suffering. PT focuses on God's supreme power of persuasion. Also, Goetz proposes that synergism between God and humans can cause some divine intervention. ${ }^{13}$

This paper introduces what I call the semiclassical cosmological argument and semiclassical theodicy. The rest of this paper describes natural science for natural theology, ST, PT, and my analysis of the kalam cosmological syllogism while comparing ST and PT.

\section{Natural science for natural theology}

Natural theology is the study of theology based on the observation and analysis of nature without appealing to any sacred text or divine revelation. For example, ST is a natural theology that coheres with modern physics. ${ }^{14}$ This section depicts various scientific theories that are important for my analysis of natural theology that focuses on the kalam cosmological syllogism and divine providence. I focus on the physics of mechanics and cosmology while including one point on the neuroscience of human will.

\subsection{Classical mechanics}

Preliminary definitions for this subsection follow:

1. Flat geometry is Euclidian geometry.

2. Newtonian absolute space is modeled with flat geometry, exists independent of anything else, and is unchangeable.

3. Newtonian absolute time exists independent of anything else and elapses at a constant rate throughout the universe.

4. Newtonian mass of an object is the object's resistance to acceleration.

5. Acceleration is a change of velocity.

Classical mechanics is an idealized model of physics derived from the concepts of absolute space, absolute time, Newtonian mass, and Newton's three laws of motion. Newton's three laws of motion follow:

1. Every object in a state of uniform motion will remain in that state of motion unless an external force acts on it.

2. Force equals mass times acceleration.

3. Every action has an equal and opposite reaction. 
Classical mechanics also implies that complete knowledge of a system enables complete knowledge of its past and future, while complete knowledge of its future is called determinism. Furthermore, classical mechanics makes extremely valuable approximations for engineering.

\subsection{Statistical mechanics, equilibrium, and nonequilibrium}

Primary subjects of statistical mechanics are equilibrium and nonequilibrium. A system in dynamic equilibrium has no macroscopic activity, but has continuous microscopic activity while opposing activities continuously reverse each other. This is called microscopic reversibility. Also, many systems in nonequilibrium approach equilibrium while also exhibiting microscopic reversibility. For example, a hypothetical system in nonequilibrium has $99 \%$ of activity that increases equilibrium and $1 \%$ of activity that decreases equilibrium while the probabilistic increase of equilibrium prevails.

Consider the example of thermodynamics in an isolated system. Thermodynamic nonequilibrium corresponds to order while equilibrium corresponds to complete disorder. Likewise, thermodynamic disorder in an isolated system probabilistically increases during the progress of time ${ }^{15}$ The increase of disorder is probabilistic because of microscopic reversibility. For example, a small fraction of thermodynamic activity decreases disorder while the rest of the activity increases disorder. Also, a thermodynamic system in complete disorder has no macroscopic activity but nonetheless exhibits microscopic reversibility.

\subsection{Special relativity (SR) and causal connection}

Preliminary definitions for this subsection follow:

1. Spacetime refers to the four-dimensional unity of the three visible spatial dimensions and the one time dimension.

2. An event is a point and all its respective phenomena in the spacetime universe. The point has four relative coordinates that are three spatial coordinates and one time coordinate.

3. A reference frame consists of an abstract coordinate system and the set of physical reference points that align the coordinate system and standardized measurements.

4. An inertial reference frame is a reference frame without acceleration.

5. An observer is located on a spacetime reference frame and makes physical measurements.

Einstein turned the world of physics upside down in 1905 when he introduced special relativity (SR). ${ }^{16} \mathrm{SR}$ is the theory of relative spacetime in the hypothetical special case of no gravity. SR is based on flat geometry and has two postulates. One, the laws of physics is identical for all inertial reference frames. Two, the speed of light is the same for all reference frames. 
Three important implications of SR for this paper are causal connection, the relativity of simultaneity, and velocity time dilation.

\subsubsection{Causal connection}

SR implies that two events are causally connected when the time interval between them is more than the spatial distance between them divided by the speed of light. Similarly, two events are causally disconnected when the time interval between them is less than the spatial distance between them divided by the speed of light.

\subsubsection{Relativity of simultaneity}

The relativity of simultaneity implies that no causally disconnected events are absolutely simultaneous. This implies the unreality of Newton's absolute time and Lorentz's preferred reference frame for a universal chronology supported by undetectable ether. ${ }^{17}$

An interesting illustration of the relativity of simultaneity is a relativistic reversal of chronology of causally disconnected events. Consider the following example with observer $A$, observer $B$, event $A$, and event $B$. Each observer and event has its own reference frame in a spacetime region that is causally disconnected from the other three reference frames. Observer A detects event B before event A. Observer B detects event A before event B. This example exemplifies the relativity of simultaneity.

\subsubsection{Velocity time dilation}

SR implies velocity time dilation. For example, an observer with a higher relative velocity has a slower progress of time compared to an observer with a lower relative velocity.

\subsection{General relativity $(G R)$}

Preliminary definitions for this subsection follow:

1. The mass of an object is a measurement based on a calculation of the object's energy and momentum, while a spring scale on Earth measures the effects of gravity on an object's mass.

2. The observable universe is the universe that is potentially observable from Earth regardless if technology permits the observation.

Einstein developed general relativity (GR) while using mind-boggling curved geometry for his famous set of field equations which generalized SR by modeling the effects of gravity. ${ }^{18}$ The curved geometry is called Riemannian geometry. Most notably, GR implies that mass causes the bending of spacetime. Consider the following examples:

1. From any inertial reference frame, the mass of a star bends the starlight of more distant stars. 
2. The mass of the Sun bends the trajectory of all planets in the Solar System. The bending causes the planetary orbits.

3. The mass of the Sun bends the space between the Sun and free falling objects. The bending causes the objects to travel from the sky to Earth.

4. Catastrophic astronomical events such as colliding black holes cause gravitational waves to ripple through spacetime.

5. An observer in stronger gravity compared to an observer in weaker gravity has a slower progress of time. This is an example of gravitational time dilation.

Also, the Riemannian geometry of GR permits an infinite number of ways to define reference frames. Furthermore, the Riemannian geometry of space implies

that an observer at any point in the observable universe sees the same spatial scale factor in every direction. For example, astrophysics confirms that the current spatial scale factor in every direction from every point in the universe is 46 billion light-years (or $10^{23}$ kilometers).

\subsection{Fundamental interactions and particle physics}

The conceptual and mathematical framework for contemporary particle physics is called quantum field theory $(Q F T) .{ }^{19}$ Most details of QFT are complicated and taught only in graduate-level physics courses, while many details of QFT are still incomplete. However, I focus only on basic concepts of QFT for my description of the observable universe.

Preliminary definitions for this subsection follow:

1. A fundamental interaction does not appear to be reducible to any more basic interaction.

2. A quantum is the minimal discrete unit of a physical object involved in interactions.

3. A quantum particle (also called a fundamental particle or elementary particle) continuously vibrates while having no substructure and likewise is not made up of other particles, while quantum vibration is technically called harmonic oscillation.

4. A quantum antiparticle (also called a fundamental antiparticle or elementary antiparticle) continuously vibrates while having no substructure and likewise is not made up of other antiparticles.

5. A quantum boson such as a photon or gluon is a quantum particle that carries energy and has integer spin and no charge.

6. A quantum fermion such as a quark or electron is a quantum particle that is matter which has mass and half-integer spin.

7. A quantum antifermion such as an antiquark or positron is a quantum antiparticle that is antimatter which has mass and half-integer spin.

8. Quantum mechanics $(Q M)$ is the mathematical description of motion and interaction for quantum particles and composite subatomic particles. 
9. A quantum system is a system composed of one or more quantum particles.

10. Zero-point energy is the lowest possible energy of a quantum system.

11. A quantized force field is a zero-point energy field composed of a single quantum particle or contiguous quantum particles.

Global consensus in the field of physics says that the four known fundamental interactions are gravitational, electromagnetic, strong, and weak. The potential for the fundamental interactions permeate the observable universe. Also, global consensus says that the electromagnetic, strong, and weak interactions are quantized force fields while the structure of gravity is disputed. For example, Einstein conjectured that gravity is caused by the continuous, forceless interaction of mass and bendable spacetime. ${ }^{20}$ However, the majority of current gravitational physicists conjecture that gravity is quantized with a miniscule scale that is beyond the direct detection of any possible technology. ${ }^{21}$ For instance, Einstein's theory of forceless gravity has no quanta and likewise has no realistic possibility of interacting with quantum particles, but a gravitational force field with zero mass coheres with QM and Einstein's field equations for GR.

If gravity is quantized, then the four fundamental interactions can properly be called the four fundamental forces, which is a commonly used term. However, if gravity is a forceless interaction, then only three of the four fundamental interactions are forces. Either way, basic QFT says that any fundamental force is a vibrating quantized field that permeates the observable universe.

Experimental physics indicates the existence of vacuum energy that permeates the observable universe. QFT says that the vacuum energy is a quantized vacuum field that reduces to a combination of all fundamental force fields. Similarly, the vacuum field is composed of vibrating particles and antiparticles with zero-point energy that permeate the observable universe. For the rest for this paper, I refer to any particle or antiparticle in the vacuum field as a vacuum particle or vacuum antiparticle.

Now I focus on particles and antiparticles. QFT describes particles and antiparticles as excited states of the vacuum. Also, every quantum fermion has a mass, a charge, and a corresponding antifermion with the same mass but opposite charge. For example, an up quark and an up antiquark have the same mass but opposite charge while an electron and a positron have the same mass but opposite charge.

Astrophysicists detect that nearly all excited states of the vacuum field are particles while only a minuscule fraction of the excited states are antiparticles. This phenomenon is called the matter-antimatter asymmetry. Regardless of the universal asymmetry, laboratory experiments produce a 1:1 ratio of matter and antimatter that coheres with the conservation of energy. For example, laboratories can convert a photon into an electron-positron pair or a proton-antiproton pair. ${ }^{22}$ This is called pair production. Also, if the newly produced matter-antimatter pair collides with each other, then they annihilate and produce one or more quantum bosons depending on the level of energy in the pair. Another interesting phenomenon is that particles sometimes spontaneously transform between particle 
and their antiparticle, and vice versa, while this transformation occurs millions of times per second before the entity decays. ${ }^{23}$

\subsubsection{Planck time and Planck length}

Natural units are units of measurement derived from the physical constants. Two important natural units for this paper are the Planck time and the Planck length.

They are the theoretically smallest units of time and length while they are derived from the constants of electromagnetic action, gravity, and the speed of light in vacuum. A Planck time is $10^{-43}$ of a second and is the time that it takes for a photon in a vacuum to travel 1 Planck length. A Planck length is $10^{-35}$ of a meter.

\subsubsection{The Copenhagen interpretation (CI) and quantum logic ( $Q L)$}

Preliminary definitions for this third-level section follow:

1. The Schrodinger equation theoretically predicts all possible quantum states for the evolution of a quantum system.

2. An observable of a quantum state such as position, momentum, time, or energy has a dynamic variable that can be measured.

3. The Schrodinger equation defines that an observable corresponds to a mathematical operator with a standard deviation and possible eigenvalues.

4. The Schrodinger equation implies that some observables have noncommutative operators, for example, position and momentum as well as time and energy.

5. A hidden variable is a theoretical variable that is undetected.

The founding fathers of QM observed that QM is drastically difference from classical mechanics. ${ }^{24}$ The majority agreed that QM is intrinsically indeterministic while the minority proposed deterministic QM supported by a theory of hidden variables. For example, the standard interpretation of QM, called the Copenhagen interpretation $(C I)$, rejects hidden-variable theory and supports indeterminism. Also, the primary tenet of the $\mathrm{CI}$ is called the uncertainty principle. This thirdlevel section describes two competing models of the uncertainty principle.

Heisenberg proposed two major implications of the uncertainty principle based on the Schrodinger equation. ${ }^{25}$ First, the evolution of any quantum system is uncertain. Second, the description of any quantum state is uncertain. The first incoheres with classical determinism while the second incoheres with classical bivalent logic based on the law of non-contradiction (LNC) by implying that a quantum state at a particular point in spacetime can simultaneously exist and not exist. However, despite popular belief, there is no unanimity within the CI that says the Schrodinger equation implies the second. For example, von Neumann in 1932 said that QM can cohere with the LNC. ${ }^{26}$ Then, Birkhoff and von Neumann in 1936 rigorously introduced quantum logic (QL) which describes the noncommutative operators of the Schrodinger equation in the context of complex mathematics and the LNC. ${ }^{27}$ For instance, they made a clear distinction between a quantum system's infinite number of potential quantum states and the actual 
quantum state at a particular point in time. Since then, QL has continued to develop. ${ }^{28}$

A popular historic misconception of the uncertainty principle involves the famous Schrodinger's cat paradox. In 1935, Schrodinger published his thought experiment that analyzed Heisenberg's QM and illustrated how a hypothetical cat can simultaneously be both dead and alive, while Schrodinger did not advocate a realistic possibility for his illustration and Heisenberg's QM. ${ }^{29}$

The history of QM implies two competing positions of the uncertainty principle. One coheres with the LNC and one is an absolute contradiction. I distinguish these two positions by calling them the bivalent uncertainty principle $(B U P)$ and the contradictory uncertainty principle $(C U P)$. For example, the BUP dispels Schrodinger's cat paradox while the CUP embraces Schrodinger's cat paradox. I support the BUP and appreciate Quine's philosophical description of a paradox. ${ }^{30}$ He defines that a paradox is an apparently successful argument with a concluding statement that seems contradictory or absurd. Rigorous analysis of the argument can resolve the paradox in two possible ways. One, careful analysis reveals that the argument is unsound while the conclusion is not completely true. Two, careful analysis reveals that the conclusion is actually true while additional explanation dispels the deceptive appearance. Also, the CUP is not considered in Quine's description of paradoxes.

Consider the pros and cons of the BUP and the CUP. The pro of the BUP is that it coheres with the LNC while the con of the BUP is that its math is more complex than the math of the CUP. Inversely, the pro of the BUP is that its math is less complex than the math of the CUP while the con of the BUP is that it violates the LNC. The heuristics for the principle of parsimony forces a choice between absolute contradiction with less mathematical complexity and the LNC with more mathematical complexity. I support the LNC over less complexity.

My primary points of this third-level section are that QM coheres with the LNC and that the evolution of any quantum system is fundamentally indeterministic. Similarly, QM indicates the existence of probabilistic causality instead of classical deterministic causality. I also clarify that quantum entanglement described in the next subsection coheres with the LNC because there is no contradiction for something to simultaneously exist in two different locations.

\subsection{Quantum entanglement and teleportation}

Preliminary definitions for this subsection follow:

1. Distant particles are separated from each other in causally disconnected locations.

2. Teleportation is the instantaneous transfer of quantum information or a quantum system from one location to another location.

Quantum entanglement with action at a distance is one of the greatest mysteries of physics. Entangled pairs of photons or electrons that are distant to each other 
exhibit simultaneous, corresponding activity. For example, Einstein, Podolsky, and Rosen proposed what is now called the EPR paradox when they analyzed quantum entanglement between distant electrons. ${ }^{31}$ The authors considered two possible explanations. First, there was an interaction between the otherwise distant particles. Second, the simultaneous, corresponding activity was causally determined by the quantum state of each particle while there was no actual interaction between the particles. They rejected the former because of SR's implications for causal connection while they favored the latter which supports causal determinism. However, the former became the standard conjecture in quantum physics. Also, the fascinating develop of quantum teleportation described below unequivocally indicates the former.

Quantum teleportation is the instantaneous transfer of quantum information from one location to a distant location. For example, quantum teleportation was first achieved in 1997 and has two major steps. ${ }^{32}$ First, a laboratory generates entangled photons. Second, the entanglement is used to instantaneously (1) destructure quantum information of a particle from the sending end of the entanglement and (2) restructure the same quantum information at the other end. This is teleportation of a quantum state from one particle to a distant particle. Also, in 2017, a ground-to-satellite laboratory had generated quantum entanglement and quantum teleportation that reached a distance of 1,400 kilometers.

Another fascinating discovery of quantum entanglement occurred in an astrophysics project that detected 30,000 pairs of entangled photons in space while the pairs have been entangled for at least 600 years. ${ }^{33}$ These photon pairs exhibited simultaneous polarized activity since the beginning of their entanglement. More recently, the astrophysicist team detected a photon pair that has been entangled for 8 billion years and currently separated by an enormous distance of 2,000 light-years (or $10^{16}$ kilometers). ${ }^{34}$ The 8 -billion-year endurance of the entanglement is older than the Sun, while the current distance of the entanglement is 23 times the distance from the Sun to its closest neighboring star.

Consensus in quantum physics supports the existence of entangled action at a distance while there is no consensus for an explanation of the entanglement. However, one and only one logically consistent conjecture for entangled action at a distance is called the $E R=E P R$ conjecture. ${ }^{35} E R=E P R$ is a pseudo acronym that refers to Einstein-Rosen bridges and the EPR paradox. The conjecture says that any pair of entangled particles (EPR) is connected by an Einstein-Rosen bridge (ER), while ER is commonly called a wormhole.

Einstein and Rosen famously proposed a GR solution that supports the possibility of wormholes which connect otherwise distant regions of spacetime. ${ }^{36}$ That is why a wormhole is sometimes called an Einstein-Rosen bridge.

Despite no scientific evidence indicating the existence of a single traversable wormhole, physicists rigorously analyze traversable wormhole theory and generally agree that some exotic matter that might not possibly exist would be needed to open the mouths at the ends of a traversable wormhole. ${ }^{37}$ However, the $\mathrm{ER}=\mathrm{EPR}$ conjecture implies that quantum wormholes in entanglement are ubiquitous in space and routinely generated in laboratories. Also, quantum 
wormholes do not require the exotically generated mouths of theoretical traversable wormholes. The ER=EPR conjecture is far from proven, while there is no other logically consistent explanation for the EPR paradox and quantum teleportation.

Consider the structure of a quantum wormhole. Any theoretical wormhole tunnels through 3D space or 4D spacetime. Either way, any wormhole tunnel has no dimensions. ${ }^{38}$ For example, entangled photons are separated by 2,000 lightyears in space while there is no spatial distance between them in the quantum wormhole. The wormhole tunnel is dimensionless while the photons indefinitely diverge from each other in space.

If the ER=EPR conjecture is true, then there is a universal potential for quantum wormhole tunnels. This implies a universal potential for no dimensions. Also, I conjecture that wormhole tunnels which have no dimensions also have no vacuum energy and no particles because vacuum energy and particles requires space. This state without dimensions, vacuum energy, and particles is what I call nothingness.

\subsection{Observers, presentism, and eternalism}

Preliminary definitions for this subsection follow:

1. Tensed time is time that progresses from the tangible present to the future while the past and future are intangible.

2. Tenseless time is time without tense while the past, present, and future are equally tangible.

This subsection briefly analyzes the contemporary debate between presentism and eternalism, while ST and PT cohere with presentism. Simple presentism says that only present objects exist and time is tensed. However, my clarified presentism introduced in this subsection says that phenomena exist only in the present and time is tensed. Also, presentism is related to the A-theory of time which typically says that time is tensed. Alternatively, eternalism says that all phenomena has always existed and will always exist while time is tenseless and tensed time is illusory. Similarly, eternalism is related to the B-theory of time that says time is tenseless while and tensed time is illusory.

Concepts of eternalism go back to the pre-Socratic philosopher Parmenides who argued against the reality of motion. The primary contemporary position of eternalism is based on SR and the Rietdijk-Putnam argument. ${ }^{39}$ I call this SR eternalism. Generalized SR eternalism follows:

1. There is no possible preferred universal chronology.

2. Objects persist through the time dimension in the same way they extend through the three spatial dimensions.

3. All past, present, and future events have always tenselessly existed in what is called the now.

4. Tensed time is illusory. 
For example, Rietdijk proposed SR eternalism and said, "A proof is given that there does not exist an event, that is not already in the past for some possible distant observer at the (our) moment that the latter is 'now' for us." ${ }^{40}$ Rietdijk refers to relativistic reversals of chronology predicted by SR and argues that any pair of distant observers would detect ubiquitous reversals of chronology. He then argues from this premise that the ubiquity of relativistic reversals of chronology implies that everything in the past, present, and future exists now. However, I will demonstrate that his premise about observers is false because there is a preferred universal chronology.

Goetz defended the A-theory of time by describing an observer in a hypothetical omnicluster of 3D wormholes. ${ }^{41}$ The omnicluster has no traversable wormholes, but the observer nonetheless detects every event in the universe as if it were a local event despite the relativity of simultaneity. I borrow from this model by analyzing two distant observers while considering entanglement and the $\mathrm{ER}=\mathrm{EPR}$ conjecture described in section 2.6. A primary point is that entangled action at a distance indicates a collapse of causal disconnection implied by SR. $\mathrm{ER}=\mathrm{EPR}$ or not $\mathrm{ER}=\mathrm{EPR}$, action at a distance is generated in physics laboratories and detected throughout space. This is an unequivocal exception to SR causality, while the ER=EPR conjecture is the only logically consistent conjecture that explains action at a distance.

Consider my following thought experiment. Two distant observers have what I call bifocal pathways. One focal pathway is called $3 D$ wormhole sight and detects events through a hypothetical omnicluster of quantum wormholes. ${ }^{42}$ The other focal pathway is called spatial-interval sight and detects events through spatial intervals that are subject to the relativity of simultaneity, which is the same as a standard SR or GR observer.

Imagine four objects called observer $A$, observer $B$, event $A$, and event $B$. Each of the four objects is located on a reference frame that is distant to the other three objects. Also, the observers A and B possess bifocal pathways.

According to the spatial-interval sight of observer A, event A occurs before event B. According to the spatial-interval sight of observer B, event B occurs before event $\mathrm{A}$. This describes a relativistic reversal of chronology for the two events and likewise exhibits the relativity of simultaneity implied by SR and GR.

However, the 3D wormhole sights of observer A and observer B are identical. They both detect event A and event B without a relative spatial interval while they detect the same chronology of event A and event B.

Imagine a four-part chronology for each observer defined as $\left(\mathrm{T}_{1}, \mathrm{~T}_{2}, \mathrm{~T}_{3}\right.$, $\left.\mathrm{T}_{4}\right)$ :

1. The spatial-interval sight of observer $A$ at $T_{1}$ detects event $A$.

2. The $3 \mathrm{D}$-wormhole sight of observer $\mathrm{A}$ at $\mathrm{T}_{2}$ detects event $\mathrm{A}$.

3. The spatial-interval sight of observer $\mathrm{A}$ at $\mathrm{T}_{3}$ detects event $\mathrm{B}$.

4. The 3D-wormhole sight of observer $\mathrm{A}$ at $\mathrm{T}_{4}$ detects event $\mathrm{B}$.

5. The spatial-interval sight of observer $B$ at $T_{1}$ detects event $B$.

6. The 3D-wormhole sight of observer $B$ at $T_{2}$ detects event $A$. 
7. The spatial-interval sight of observer $B$ at $T_{3}$ detects event $A$.

8. The 3D-wormhole sight of observer $\mathrm{B}$ at $\mathrm{T}_{4}$ detects event $\mathrm{B}$.



Figure 1. Schematic illustration of observer $A(O A)$ at $\left(T_{1}, T_{2}, T_{3}, T_{4}\right)$; observer $B$ $(\mathrm{OB})$ at $\left(\mathrm{T}_{1}, \mathrm{~T}_{2}, \mathrm{~T}_{3}, \mathrm{~T}_{4}\right)$; event $\mathrm{A}(\mathrm{EA})$; and event $\mathrm{B}$ (EB). Curved lines represent spatial-interval sight; straight lines represent teleportative sight.

The spatial-interval sight exhibits no transitivity as implied by the relativity of simultaneity. For example, the spatial-interval sight of OA detects EA before EB while the spatial-interval sight of $\mathrm{OB}$ detects $\mathrm{EB}$ before EA.

Alternatively, the 3D-wormhole sight exhibits transitivity. For example, the $3 \mathrm{D}$ wormhole sights of $\mathrm{OA}$ and $\mathrm{OB}$ detect the exact same chronology of EA and $\mathrm{EB}$.

Furthermore, when observer A and observer B use spatial-interval sight, they detect an infinite set of relative reversals of chronology in the observable universe. ${ }^{43}$ However, when observer A and observer B use 3D wormhole site, they see the exact same chronology of the entire observable universe.

Moreover, an observer with 4D sight would see the same chronology as an observer with 3D sight. However, 4D sight is logically possible only if past and future phenomena exist in a universe where objects persist in the time dimension in the same way they extend in the spatial dimensions.

The long-standing premise of no possible preferred universal chronology is false because of ubiquitous action at a distance and observers with 3Dwormhole sight, while 3D-wormhole sight is the preferred focal pathway for a universal chronology. Similarly, the SR unity of spacetime does not imply that objects persist through the time dimension in the same way they extend through the spatial dimensions. Also, all observation of cause and effect, especially irreversible reaction in physics and chemistry, coheres with presentism.

\subsection{Composite systems}

I simply define that a composite system is a system composed of two or more unentangled elementary particles. For example, gluons bind subatomic composite particles and also atoms; while chemical bonds bind systems such as molecules and living organisms. On the astronomical scale, gravity binds subatomic particles 
and molecules into systems such as interstellar clouds, stars, galaxies, and clusters of galaxies. Another interesting phenomenon is called self-organization.

Self-organization is a process that involves numerous interactions among local-level components of a system that cause the emergence of a global-level pattern in spacetime. This describes synergistic emergence while the science of self-organization indicates that synergistic emergence is ubiquitous in nature and society. ${ }^{44}$ Similarly, synergism by definition is the interaction of two or more components that produce an emergent effect which is greater than the sum of the components' separate effects. I also clarify that self-organization is dependent on the environment such as space and respective mediums.

\subsection{Physical cosmology}

Hubble made the fascinating discovery that most galaxies are moving away from each other while the observable universe is expanding. ${ }^{45}$ One way to imagine this expansion is to partially blow up a balloon, speckle it with spots, and then continue to blow up the balloon while watching all the dots move away from each other. However, this imagery is oversimplified because the surface of a balloon is a 2D Euclidian plane while the space of the expanding universe is 3D Riemannian manifold which is beyond illustration.

Physical cosmology models measurements of the observable universe and then extrapolates from the model to make conjectures about the past and future of the universe. The Standard Model of cosmology is called the Lambda cold dark model $(\Lambda C D M)$ model, which is a Big Bang cosmology. ${ }^{46}$

Backwards extrapolation of the expanding universe results in an original hot singularity, that is, a dimensionless point with infinite density and likewise infinite curvature. However, physicists are undecided if the universe began with a hot singularity because we lack the knowledge needed to describe such a hot dense state. Nonetheless, if there was an original hot singularity, it existed during the Planck epoch and endured 1 Planck time.

There is also no consensus for a model that explains the origin of quantum particles. Major debate focuses on whether the universe had a finite past or an infinite past. A cosmology with a finite past means that the universe had a foremost origin while a cosmology with an infinite past means that the universe has always exists with no foremost origin.

Mithani and Vilenkin analyzed three different categories of cosmologies with an infinite past and found major problems with all of them while concluding that the universe probably had a finite past. ${ }^{47}$ The authors looked at eternal inflation with no beginning, cyclic cosmology, and the emergent universe.

\subsubsection{Eternal inflation}

$\Lambda \mathrm{CDM}$ cosmology indicates a brief period of the observable universe exhibiting rapid expansion called inflation. ${ }^{48}$ Eternal inflation proposes that the observable universe is a mere pocket universe in a multiverse that never ceases to inflate and generate new pocket universes, while vacuum energy and quantum fluctuations perpetuate the inflation. ${ }^{49}$ Also, inflationary multiverse theory uses Riemannian 
geometry that can support an infinite set of pocket universes while each pocket is modeled as a manifold. Furthermore, some propose that eternal inflation has an infinite past. However, the geometry of an expanding multiverse indicates that eternal inflation has a finite past. ${ }^{50}$

\subsubsection{Cyclic cosmology}

Contemporary cyclic cosmologies propose an infinite past series of a big bang followed by expansion and then contraction into a crunch that transitions into the next big bang. However, entropy would increase with each cycle while the universe would face thermal death unless the volume of the universe increases with each cycle. Alternatively, if the volume of the universe increases with each cycle, then the cyclic universe would face the same geometric problem as eternal inflation which requires a finite past. ${ }^{51}$

\subsubsection{Emergent universe cosmology}

Emergent universe cosmology can be imagined as a static seed that existed since the infinite past and endured until the finite past when it began to inflate. For example, the Riemannian geometry of the static seed approaches the limit of negative infinity. However, QM indicates instability that would not permit the static seed to endure the infinite past. ${ }^{52}$

\subsubsection{A spacetime universe from nothingness}

Can a spacetime universe originate from nothingness without a cause?

For example, Krauss proposes that nothingness is inherently unstable while the observable universe and other spacetime universes originated from unstable nothingness that generated quantum fluctuations. ${ }^{53}$ If he is correct about the inherent instability of nothingness, then unstable nothingness and quantum fluctuations have always existed and likewise have existed since the infinite past.

Consider any possible quantum fluctuation with a prior probability greater than zero. For example, Penrose estimated that the origin of low entropy at the beginning of a big bang similar to the origin of the observable universe has an absurdly minuscule probability of $1 / 10^{\left(10^{\wedge} 123\right)} \cdot{ }^{54}$ Now, imagine an infinite set of trials with a probability of $1 / 10^{\left(10^{\wedge} 123\right)}$ for generating a similar big bang. The trials would result in an infinite number of similar big bangs. ${ }^{55}$ However, section 4 describes problems with erroneous use of infinity in arithmetic operations. Also, consider the probability of $1 / 10^{\left(10^{\wedge} 123\right)}$ and a finite number of trials. For example, Goetz calculated that $10 \times 10^{\left(10^{\wedge} 123\right)}$ trials would result in 1 or more similar big bangs with a confidence level of $99.995 \% .{ }^{56}$ For instance, imagine the generation of vacuum energy with the same physical constants that exist in the observable universe, while every vibration cycle of the energy is a possible fluctuation. Likewise, $10 \times 10^{\left(10^{\wedge} 123\right)}$ vibration cycles would likely generate 1 or more similar big bangs. Furthermore, if there is an infinite set of possible combinations of physics constants, then the probability for randomly generating any particular combination of physical constants approaches 0 . 


\subsubsection{Semiclassical cosmology}

ST cosmology proposes an internally static manifold has tenselessly existed since the infinite past and likewise contains no quantum vibrations or fluctuations. ${ }^{57}$ Also, the internally static manifold can generate an external quantum system that becomes a tensed spacetime manifold, such as the observable universe. ${ }^{58}$ Furthermore, the generation of a new spacetime manifold is creation from nothing because it is external to any other manifold. For example, section 2.6 describes that a state of no dimensions and quantum systems is nothingness. Additionally, QM indicates that any manifold with 3D space contains quantum vibrations or fluctuations, so I conjecture that an internally static manifold would have less than three spatial dimensions.

\subsection{Neuroscience and human will}

ST and PT propose that humans possess free will, so I briefly touch on the neuroscience of human will. First of all, the concept of free will is hard to define, while I appreciate Lavazza's focus that says an agent with free will exhibits free choices and voluntary behavior as opposed to always exhibiting automatic behavior. ${ }^{59}$

Various neuroscience studies have suggested that all human decisions are made in the subconscious while all decisions and behavior are automatic responses to the environment. ${ }^{60}$ This can compare to Newton's third law of motion. For example, every human reaction is caused by an equal and opposite action. However, other neuroscience studies indicate that humans sometimes veto their subconscious decisions. ${ }^{61}$ Their decision making and behavior is primarily automatic yet not completely automatic. This indicates that humans possess partial free will. Similarly, this permits humans some freedom in how they choose to train themselves while developing their morals and skills.

\section{Summary of semiclassical theism (ST) and process theism (PT)}

\subsection{ST}

Goetz's introduction to ST contains complex concepts from physics, philosophy, and religion. ${ }^{62} \mathrm{~A}$ summary follows:

1. God originally exists in infinite tenseless eternity while possessing the attributes of love, perception, and force.

2. Everything in tenseless eternity is completely simultaneous with no beginning boundary and no ending boundary.

3. Everything that is tenseless is necessarily everlasting, so the primary divine attributes are everlasting love, perception, and force.

4. God lovingly created the observable universe to lovingly create and relate to rational agents of authority, for example, humans.

5. God created the observable universe and any other spacetime universes out of nothing in the finite past while constrained by the possibilities of physics. 
6. A spacetime universe is tensed and external to tenseless eternity.

7. Riemannian geometry is the mathematical basis for general relativity, inflationary multiverse models, and the semiclassical relationship between infinite tenseless eternity and spacetime universes.

8. The mechanics of a spacetime universe are governed by the bendability of spacetime and the probabilistic causality of vacuum energy, quantum particles, nuclear reactions, chemical reactions, thermodynamics, and fluid dynamics.

9. A hypothetical omnicluster of 3D wormholes enables God's omniperception of and omnipresence in tensed creation.

10. God's everlasting force that can create a spacetime universe out of nothing cannot meticulously control the particles of the creation, but synergy between God and created agents can exhibit limited intervention in the creation that is subject to the possibilities of physics.

\subsection{PT}

PT is based on process philosophy pioneered by Alfred North Whitehead. For example, the primary tenet of process philosophy is that all existence is in some way dynamic and likewise tensed. ${ }^{63}$

Mainstream elements of process theology follow:

1. God is necessarily and fully involved in and affected by tensed processes.

2. God is in some ways eternal, immutable, and impassible; while God is in some ways tensed, mutable, and passible.

3 . God is the supreme creative power and possesses everlasting love and complete knowledge of the present.

4. God acts by persuasion instead of by coercion.

5. God created the observable universe out of preexisting creation.

6 . The process of creating spacetime universes out a preexisting creation had no foremost beginning.

7. The spacetime universe is in some sense part of God, which is the doctrine of panentheism.

I also note the heterodox process theist position of Edwards. ${ }^{64}$ The heterodox process theist position states that God created a beginningless series of spacetime universes out of nothing.

One way or another, process philosophy, PT, and heterodox PT imply a beginningless series of spacetime universes while all spacetime universes possess tensed quantum vibrations. Also, mathematical logic implies that no foremost beginning of tensed quantum vibrations imply an infinite past series of tensed quantum vibrations. 


\section{The semiclassical cosmological argument}

The syllogism of the kalam cosmological argument follows:

Major premise: Whatever begins to exist had a cause.

Minor premise: The physical universe began to exist.

Conclusion: Therefore, the physical universe had a cause. ${ }^{65}$

For the purpose of this paper, I modify the minor premise and conclusion to focus on tensed Planck time intervals. My clarified adaptation of the kalam cosmological syllogism follows:

Major premise: Whatever begins to exist had a cause.

Minor premise: Tensed Planck time intervals foremostly began to exist.

Conclusion: Therefore, the foremost beginning of tensed Planck time intervals had a cause.

The kalam cosmological syllogism implies that an uncaused entity caused the beginning of tensed Planck time intervals. In other words, Proposition 3 follows:

Proposition 3: The kalam cosmological syllogism implies that an uncaused entity caused the foremost beginning of tensed Planck time intervals.

The kalam cosmological argument concludes that the uncaused entity is called God and possesses the traditionally defined uncaused attributes of omnipotence, omniscience, and omnibenevolence. ${ }^{66}$ This leads to intense discussion about the problem of evil. However, proof for a foremost creation out of nothing does not necessitate belief that God possesses the traditional divine attributes of omnipotence, omniscience, and omnibenevolence. For example, minimal tautological implications of the kalam cosmological syllogism are that an uncreated entity with consciousness and force had created a tensed universe out of nothing. Also, the uncreated entity with consciousness and force is inexhaustible and likewise everlasting. This argument does not support belief in the traditional divine attributes, but it does support belief in the uncreated creator of the tensed universe who I call God.

Goetz concludes that God possesses the uncaused attributes of everlasting force, perception, and love; while the everlasting force of God cannot possibly meticulously control creation. ${ }^{67}$ In this position, there is no need to resolve the problem of evil. These different definitions of divine attributes distinguish the semiclassical cosmological argument from the traditional kalam cosmological argument. 
The syllogism is flawlessly valid. Objecting to the conclusion requires objecting to at least one of the premises.

Scientific observation indicates the major premise that states that "Whatever begins to exist had a cause." Likewise, objecting to the major premise has the consequence of objecting to the basics of modern science. However, some object to the major premise. ${ }^{68}$ I agree that the major premise is not logically necessary. For example, all experimental physics indicates that everything with a beginning had a cause, and it is reasonable to make the inductive conclusion that everything with a beginning indeed had a cause. But that does not absolutely prove that there are no exceptions to the scientific norm that something with a beginning had a cause. For instance, inductive reasoning is the basis of the scientific method and scientific proof. Likewise, the strongest cases of scientific proof are inductive and cannot possibly result in absolute proof of anything.

Noting the inevident logical possibility that something could begin without a cause, there is no positive evidence that something had a beginning without a cause and there is strong scientific evidence that indicates that everything with a beginning had a cause. Also, process theists accept the basics of modern science, while additional defense of the major premise is beyond the scope of this paper.

However, the minor premise that states that "Tensed Planck time intervals foremostly began to exist" is controversial in philosophy and science. Also, process theists object to the minor premise.

One way to object to the minor premise is to propose that tensed time never existed and is a mere illusion as concluded by the positions of eternalism and B-theory. However, section 2.7 (1) demonstrates that SR does not imply eternalism and B-theory, (2) argues against eternalism and B-theory, and (3) argues for presentism and A-theory. Also, ST and PT object to any position that states that tensed time is a mere illusion, while additional support for the reality of tensed time is beyond the scope of this paper.

Despite ST and PT agreeing on the reality of tensed time, their disagreement about the minor premise is a qualitative difference between ST and PT. For example, process philosophy and PT imply a past infinite series of tensed particle vibrations. ${ }^{69}$ Similarly, a past infinite series of tensed particle vibrations implies a past infinite series of tensed Planck time intervals. Therefore, PP and PT object to the minor premise. However, Proposition 4 is a sub premise for the minor premise and is a necessary proposition:

Proposition 4: A past infinite series of tensed Planck time intervals is logically impossible.

Proposition 4 is necessarily true. Any cosmology that implies a past infinite series of tensed Planck time intervals is disproven and likewise false. In other words, any cosmology that implies tensed cause and effect of physical processes with no foremost beginning is false. Likewise, this cosmological argument implies that the $\mathrm{PP}$ tenet of a dynamic universe existing with no foremost beginning is logically impossible. 
Perhaps one might object to Proposition 4 because of misunderstanding the concept of infinity. For example, misuse of number theory in the case of the term infinity and its symbol $\infty$ abounds. For instance, the correct use of infinity includes geometry, set theory, and limits. Also, Propositions 5-7 exemplify the correct use of infinity:

Proposition 5: Any line segment has an infinite number of distinct points.

Proposition 6: The countable set of natural numbers has an infinite number of natural numbers.

Proposition 7: The infinite set of real numbers and the infinite set of rational numbers are infinite sets with different sizes.

However, some people misunderstand the difference between actual infinity and potential infinity. The term potential infinity refers to no natural number but to a never ending process. ${ }^{70}$ For example, the process of individually counting each natural number in the countably infinite set of natural numbers would never end. In other words, completing the process of successively counting individual natural numbers is logically impossible. Also, any erroneous use of $\mp \infty$ in arithmetic operations results in a hypothetical calculation that is undefined or logically impossible.

Zeno reminds us that abstract geometry implies that any interval is subject to infinite divisibility. ${ }^{71}$ However, the abstract infinite divisibility has no relevance to measurements in the context of quantum intervals, such as Planck lengths and Planck times.

Consider inflationary multiverse models pioneered by Linde. ${ }^{72}$ Linde concedes to the argument recounted in section 2.9.1 that implies an original boundary for an inflationary multiverse, yet Linde proposes a past infinite series of multiverse boundaries. The model of a past infinite series of multiverse boundaries is ultimately a model of no foremost beginning. For example, the Riemannian geometry of general relativity permits the possibility for an infinite number of Riemannian manifolds with infinite size. However, a past infinite series of tensed multiverse boundaries implies a past infinite series of tensed Planck time intervals that are not permitted by the correct use of number theory and Proposition 4. Any inflationary multiverse model with no foremost boundary is mathematically impossible unless tensed cause and effect is unreal and likewise illusory.

Alternatively, an internally static manifold has no foremost beginning, no end, and no progress of time. For example, everything in an internally static manifold is tenseless, continuous, and simultaneous. Also, an internally static manifold has a past infinite duration of time relative to any point in any spacetime universe. Furthermore, an internally static manifold logically coheres with number theory because it has no progress of time.

ST states that God originally exists in tenseless eternity with everlasting love, perception, and force. ${ }^{73}$ Also, Goetz notes that tenseless eternity could 
include the infinite loving relationships of the Trinity. ${ }^{74}$ This position of tenseless eternity coheres with an internally static manifold that is infinite, continuous, and simultaneous.

ST also states that God created the foremost spacetime universe out of nothing in the finite past while constrained by the possibilities of physics; and all spacetime universes are external to tenseless eternity. This position coheres with the kalam cosmological syllogism. Furthermore, the creation of a spacetime universe out of nothing could begin with the generation of the physical constants and vacuum energy followed by one or more vacuum fluctuations. Then, God's everlasting attributes maintained perception of and presence in the spacetime universe, but by no means could God meticulously control the probabilistic processes of the universe.

Finally, Goetz notes that God lovingly created rational progeny with free will and the responsibility of authority, such as humans; while God pursues loving relationships with the progeny. ${ }^{75}$ This implies that God created the progeny with evolutionary processes that are beyond the possibility of meticulous control. Also, section 2.10 describes Lavazza's conjecture that neuroscience indicates that humans possess partial free will despite the primary automation of the human nervous system. Similarly, the natural theology of ST includes the reality of human free will within nomological limits.

\section{Conclusion}

Based on the positions of divine providence described in section 1, I define three top types of divine providence that are meticulous providence, semi-meticulous providence, and supreme providence.

First, I use Sanders's definition for meticulous providence. For example, meticulous providence implies meticulous sovereignty in that God one way or another selects the outcome of everything in creation. ${ }^{76}$ Second, I modify Sanders's definition of general providence by calling it semi-meticulous providence because meticulous providence was nomologically possible except that God voluntarily decided to allow freewill creatures to make decisions that are not selected by God. ${ }^{77}$ Semi-meticulous providence also implies semi-meticulous sovereignty. Third, I defined supreme providence that implies supreme sovereignty and that God lovingly governs the universe while meticulous providence is nomologically impossible.

A position of supreme providence is a reasonable response to the problem of evil and the kalam cosmological syllogism. Analysis of objections to supreme providence based on various sacred traditions are important and beyond the scope of this paper. Other important analysis beyond the scope of this paper includes the fate of postmortem humans.

Among the positions of supreme providence, ST is logically possible and coheres with modern physics while the section 4 cosmological argument implies that the PT position of no foremost creation of dynamic processes is logically impossible. Alternatively, the previously described ST combined with Relative- 
Social Trinitarianism implies simultaneously infinite loving relationships with no foremost beginning and no end. Also, dynamic loving activity has no end.

\section{Acknowledgements}

I thank Jeremy Goetz, Trent Dougherty, John Sanders, Thomas Jay Oord, Mark C. Murphy, Graham Oppy, and various anonymous reviewers for reading earlier versions of my paper. Any remaining errors are mine.

\section{Declaration of interest}

This research had no conflicts of interest and no financial support.

${ }^{1}$ John Milton, Paradise Lost, 2nd edition (1674), 1.24-26, http://www.dartmouth.edu/ milton/reading_room/pl/intro/text.shtml.

2 Trent Dougherty, "Recent Work on the Problem of Evil," Analysis 71, no. 3 (2011): 560-73.

${ }^{3}$ James Goetz, "Semiclassical Theism and the Passage of Planck Times," Theology and Science 14, no. 3 (2016): 334-35; Thomas Aquinas, Summa Theologica, trans. Fathers of the English Dominican Province (1947): 1.25.4, https://www.ccel.org/ccel/aquinas/summa.FP_Q25_A4.html.

${ }^{4}$ John Sanders, The God Who Risks: A Theology of Divine Providence, 2nd edition (Downers Grove: IVP Academic, 2007), 11-17.

${ }^{5}$ Charles Hartshorne, The Divine Relativity: A Social Conception of God (New Haven: Yale University Press, 1948); Charles Hartshorne, Omnipotence and Other Theological Mistakes (Albany: State University of New York Press, 1984).

${ }^{6}$ Rem B. Edwards, "How Process Theology Can Affirm Creation Ex Nihilo," Process Studies 29, no. 1 (2000): 77-96.

${ }^{7}$ David Ray Griffin, "Creation Out of Chaos and the Problem of Evil," in Encountering Evil: Live Options in Theodicy, 2nd edition, ed. Stephen T. Davis (Louisville: Westminster John Knox, 2001), 108-44.

${ }^{8}$ John B. Cobb, Jr., A Natural Christian Theology: Based on the Thought of Alfred North Whitehead, 2nd edition (Louisville: Westminster John Knox, 2007). ${ }^{9}$ Thomas Jay Oord, The Nature of Love: A Theology (Atlanta: Chalice, 2010); Thomas Jay Oord, "God Always Creates out of Creation in Love: Creatio Ex Creatione a Natura Amoris," in Theologies of Creation: Creatio Ex Nihilo and Its New Rivals, ed. Thomas Jay Oord (New York: Routledge, 2014), 109-22; Thomas Jay Oord, The Uncontrolling Love of God: An Open and Relational Account of Providence (Downers Grove: IVP Academic, 2015); Thomas Jay Oord, "An Essential Kenosis View," in God and the Problem of Evil: Five Views, eds. Chad Meister and James K. Drew, Jr. (Downers Grove: IVP Academic, 2017), 77-98.

${ }^{10}$ Goetz, "Semiclassical Theism."

${ }^{11}$ Plato, Timaeus, trans. Benjamin Jowett (360 BCE), http://classics.mit.edu/Plato/timaeus.html.

${ }^{12}$ Goetz, "Semiclassical Theism," 333-34. 
${ }^{13}$ Ibid, 334.

${ }^{14}$ Goetz, "Semiclassical Theism."

15 The second law of thermodynamics enshrines this observation.

${ }^{16}$ Albert Einstein, Relativity: The Special and the General Theory, trans. Robert

W. Lawson (New York: Three Rivers Press, 1961).

${ }^{17}$ For Lorentz's preferred reference frame for a universal chronology, see Hendrik A. Lorentz, "Electromagnetic Phenomena in a System Moving with any Velocity Smaller than that of Light," Proceedings of the Royal Netherlands Academy of Arts and Science 6 (1904): 809-31.

${ }^{18}$ Einstein, Relativity.

${ }^{19}$ David Wallace, "The Quantum Theory of Fields," in The Routledge Companion to the Philosophy of Physics, eds. Eleanor Knox and Alastair Wilson (New York, Routledge, forthcoming), http://philsci-archive.pitt.edu/15296/.

${ }^{20}$ Einstein, Relativity.

${ }^{21}$ Freeman Dyson, "Is a Graviton Detectable?" 2012, https://publications.ias.edu/sites/default/files/poincare2012.pdf.

${ }^{22}$ The proton is made of three quarks bound by gluons while the antiproton is made of three antiquarks bound by gluons

23 "The Matter-Antimatter Asymmetry Problem," CERN, accessed June 10, 2019, https://home.cern/science/physics/matter-antimatter-asymmetry-problem.

${ }^{24}$ Werner Heisenberg, The Physical Principles of the Quantum Theory, trans. Carl Eckart and Frank C. Hoyt (Chicago: University of Chicago Press, 1930).

${ }^{25}$ Ibid.

${ }^{26}$ John von Neumann, Mathematische Grundlagen der Quantenmechanik (Berlin: Springer, 1932).

${ }^{27}$ Garrett Birkhoff and John von Neumann, "The Logic of Quantum Mechanics," Annals of Mathematics 37, no. 4 (1936): 823-43.

${ }^{28}$ Christian de Ronde, Graciela Domenech, and Hector Freytes, "Quantum Logic in Historical and Philosophical Perspective," Internet Encyclopedia of

Philosophy, accessed June 10, 2019, https://www.iep.utm.edu/qu-logic/; Masanao Ozawa, "Quantum Reality and Measurement: A Quantum Logical Approach," Foundations of Physics 41, no. 3 (2011): 592-607; Cyril Branciard, "ErrorTradeoff and Error-Disturbance Relations for Incompatible Quantum Measurements," Proceedings of the National Academy of Sciences of the United States of America 110, no. 17 (2013): 6742-47; Masanao Ozawa, "Soundness and Completeness of Quantum Root-Mean-Square Errors," npj Quantum Information 5 (2019): 1.

${ }^{29}$ John D. Trimmer, "The Present Situation in Quantum Mechanics: A Translation of Schrodinger's 'Cat Paradox' Paper," Proceedings of the American Philosophical Society 124, no. 5 (1980): 323-38.

${ }^{30}$ Willard Van Orman Quine, The Ways of Paradox: And Other Essays, 2nd edition (Cambridge: Harvard University Press, 1976), 1-18.

${ }^{31}$ Albert Einstein, Boris Podolsky, and Nathan Rosen, "Can Quantum-Mechanical Description of Physical Reality Be Considered Complete?" Physical Review 47, no. 10 (1935): 777-80. 
${ }^{32}$ Nicolas Gisin, "Quantum-Teleportation Experiments Turn 20," Nature 552, no. 7683 (2017): 42-43.

${ }^{33}$ Johannes Handsteiner et al., "Cosmic Bell Test: Measurement Settings from Milky Way Stars," Physical Review Letters 118, no. 6 (2017): 060401.

${ }^{34}$ Dominik Rauch et al., "Cosmic Bell Test Using Random Measurement Settings from High-Redshift Quasars," Physical Review Letters 121, no. 8 (2018): 080403.

${ }^{35}$ Juan Maldacena and Leonard Susskind, "Cool Horizons for Entangled Black Holes," Fortschritte der Physik 61, no. 9 (2013): 781-811; Kristan Jensen and Andreas Karch, "Holographic Dual of an Einstein-Podolsky-Rosen Pair Has a Wormhole," Physical Review Letters 111, no. 20 (2013): 211602; Leonard Susskind, "ER=EPR, GHZ, and the Consistency of Quantum Measurements," Fortschritte der Physik 64, no. 1 (2016): 72-83; ChunJun Cao, Sean M. Carroll, and Spyridon Michalakis, "Space from Hilbert Space: Recovering Geometry from Bulk Entanglement," Physical Review D, 95, no. 2 (2017), 024031; Leonard Susskind, "Copenhagen vs Everett, Teleportation, and ER=EPR," Fortschritte der Physik 64, no. 6-7 (2016): 551-6; Leonard Susskind and Ying Zhao, "Teleportation through the Wormhole," Physical Review D 98, no. 4 (2018): 046016. Also, search "ER=EPR" at Google Scholar since 2013 for more citations. ${ }^{36}$ Albert Einstein and Nathan Rosen, "The Particle Problem in the General Theory of Relativity," Physical Review 48, no. 1 (1935): 73-78.

${ }^{37}$ Oliver James et al., "Visualizing Interstellar's Wormhole," American Journal of Physics 83 no. 6 (2015): 486-99.

${ }^{38}$ Traversable wormhole throats at the two mouths of the wormholes have spatial dimensions, but the tunnel of a traversable wormhole or a quantum wormhole has no dimensions.

${ }^{39}$ C. W. Rietdijk, "A Rigorous Proof of Determinism Derived from the Special Theory of Relativity," Philosophy of Science 33, no. 4 (1966): 341-44; Hilary Putnam, "Time and Physical Geometry," The Journal of Philosophy 64, no. 8 (1967): 240-247; Robin Le Poidevin, Change, Cause and Contradiction: A Defence of the Tenseless Theory of Time (London: Palgrave Macmillan, 1991); Huw Price, Time's Arrow and Archimedes' Point: New Directions for the Physics of Time (Oxford: Oxford University Press, 1997); Steven F. Savitt, "There's No Time like the Present (in Minkowski Spacetime)," Philosophy of Science 67 (Supplement 2000): 563-74; Roger Penrose, The Emperor's New Mind: Concerning Computers, Minds, and the Laws of Physics (Oxford: Oxford University Press, 2002), 299-305; Michael C. Rea, "Time, Space-Time, and Persistence," in The Oxford Handbook of Metaphysics, eds. Michael J. Loux and Dean W. Zimmerman (Oxford: Oxford University Press, 2005), 240-80; Christian Wuthrich, "The Fate of Presentism in Modern Physics," in New Papers on the Present: Focus on Presentism, eds. Roberto Ciuni, Kristie Miller, Giuliano Torrengo (Munich: Philosophia, 2011) 92-133.

${ }^{40}$ Rietdijk, "A Rigorous Proof," 341.

${ }^{41}$ Goetz, "Semiclassical Theism," 331-32.

${ }^{42}$ I emphasize that the omnicluster of quantum wormholes is hypothetical while the widespread cases of entanglement are not an omnicluster. 
${ }^{43}$ One might misunderstand what I mean by an infinite set of relative reversals of chronology. I clarify that any interval in an abstract coordinate system is infinitely divisible and likewise has an infinite set of points, while every point corresponds to an event in the relative spacetime coordinate system.

${ }^{44}$ Herman Haken, founding ed. Springer Series in Synergetics, 119 vols. (Cham: Springer (1974-); Herman Haken, "Self-organization," Scholarpedia 3, no. 8 (2008): 1401, https://doi.org/10.4249/scholarpedia.1401.

${ }^{45}$ Edwin Hubble, "A Relation between Distance and Radial Velocity among Extra-Galactic Nebulae," Proceedings of the National Academy of Sciences 15, no. 3 (1929) 168-73.

46 " $\Lambda$ CDM Graphical History," NASA, last updated August 24, 2016, https://lambda.gsfc.nasa.gov/education/graphic_history/index.cfm.

${ }^{47}$ Audrey Mithani and Alexander Vilenkin, "Did the Universe Have a Beginning?" (paper presented at the Xth International Conference on Gravitation, Astrophysics and Cosmology, Quy-Nhon, Vietnam, December 17-22, 2011). 48 " $\Lambda$ CDM Graphical History."

${ }^{49}$ Alan H. Guth, "Eternal Inflation and Its Implications," Journal of Physics A: Mathematical and Theoretical 40, no. 25 (2007): 6811-26.

${ }^{50}$ Mithani and Vilenkin, "Did the Universe Have a Beginning?"

${ }^{51}$ Ibid.

52 Ibid.

${ }^{53}$ Lawrence M. Krauss, A Universe from Nothing: Why There Is Something Rather than Nothing (New York: Free Press, 2012).

${ }^{54}$ Penrose, The Emperor's New Mind, 440-49.

${ }^{55}$ James Goetz, "Classical Probability, Shakespearean Sonnets, and Multiverse Hypotheses," archived 2006 at International Society for Complexity, Information, and Design, https://philpapers.org/archive/GOECPS.pdf.

${ }^{56}$ Ibid.

${ }^{57}$ Goetz, "Semiclassical Theism," 329-31.

${ }^{58}$ Ibid, 333.

${ }^{59}$ Andrea Lavazza, "Free Will and Neuroscience: From Explaining Freedom Away to New Ways of Operationalizing and Measuring It," Frontiers in Human Neuroscience, (2016), https://doi.org/10.3389/fnhum.2016.00262.

${ }^{60}$ Ibid.

${ }^{61}$ Ibid.

${ }^{62}$ Goetz, "Semiclassical Theism."

${ }^{63}$ Alfred North Whitehead, The Concept of Nature (Cambridge: Cambridge University Press, 1920).

${ }^{64}$ Edwards, "How Process Theology Can Affirm Creation Ex Nihilo."

${ }^{65}$ William Lane Craig and James D. Sinclair, "The Kalam Cosmological Argument," in The Blackwell Companion to Natural Theology, eds. William Lane Craig and J.P. Moreland (Oxford: John Wiley \& Sons, 2009), 101-201.

${ }^{66}$ Ibid.

${ }^{67}$ Goetz, "Semiclassical Theism."

${ }^{68}$ Graham Oppy, "Arguing about the Kalam Cosmological Argument," Philo 5, no. 1 (2002): 34-61. 
${ }^{69}$ See section 3.2.

${ }^{70}$ Aristotle, Metaphysics, trans. William David Ross, (350 BCE), 9.6, http://classics.mit.edu/Aristotle/metaphysics.html.

${ }^{71}$ Aristotle, Physics. trans. R. P. Hardie and R. K. Gaye, (350 BCE), http://classics.mit.edu/Aristotle/physics.html.

${ }^{72}$ Andrei Linde, "Inflationary Cosmology after Planck," in Post-Planck Cosmology: Lecture Notes of the Les Houches Summer School: Volume 100: July 2013, eds. Cedric Deffayet, Patrick Peter, Benjamin Wandelt, Matias Zaldarriaga, and Leticia F. Cugliandolo (Oxford: Oxford University Press, 2015), 231-316. ${ }^{73}$ See section 3.1.

${ }^{74}$ Goetz, "Semiclassical Theism," 330. Also, see James Goetz, "Identical Legal Entities and the Trinity: Relative-Social Trinitarianism," Journal of Analytic Theology 4 (2016).

${ }^{75}$ See section 3.1.

${ }^{76}$ See section 1 for Sanders's definition of meticulous providence.

${ }^{77}$ See section 1 for Sanders's definition of general providence. 